\title{
BMJ Open The direct and indirect impact of comorbidity on the survival of patients with non-small cell lung cancer: a combination of survival, staging and resection models with missing measurements in covariates
}

\author{
Maria lachina, ${ }^{1}$ Anders Green, ${ }^{2}$ Erik Jakobsen ${ }^{2,3}$
}

To cite: Iachina M, Green A, Jakobsen $\mathrm{E}$. The direct and indirect impact of comorbidity on the survival of patients with nonsmall cell lung cancer: a combination of survival, staging and resection models with missing measurements in covariates. BMJ Open 2014:4:e003846.

doi:10.1136/bmjopen-2013003846

- Prepublication history for this paper is available online. To view these files please visit the journal online (http://dx.doi.org/10.1136/ bmjopen-2013-003846).

Received 22 August 2013 Accepted 14 January 2014

CrossMark

For numbered affiliations see end of article.

Correspondence to Dr Maria lachina; maria.iachina@rsyd.dk

\section{ABSTRACT}

Objective: To examine the direct and indirect impact of comorbidity on the survival.

Design: A historical cohort study.

Setting: Denmark.

Participants: All patients with non-small cell lung cancer who were registered in the Danish Lung Cancer Registry in 2010.

Main outcome measures: The influence of comorbidity on stage misclassification, probability of resection and survival.

Results: It was estimated that the comorbidity influences the probability of resection with OR 0.65 and $95 \%$ credible interval $(0.54 ; 0.79)$, the staging process with OR 1.08 and $95 \%$ credible interval $(0.96$; 1.20 ), and the survival process with HR 1.08 and $95 \%$ credible interval $(1.02 ; 1.14)$.

Conclusions: We found that comorbidity has a significant indirect effect on survival mediated by the resection process and a slightly direct effect on mortality.

\section{INTRODUCTION}

Primary lung cancer is one of the most common cancers in Denmark with more than 4000 new cases/year. The prognosis for patients with lung cancer is poor with crude 5-year survival proportions of approximately $10-12 \%$. However, there is evidence of some improvement in patient mortality in most recent years. ${ }^{1}$ Approximately $90 \%$ of lung cancers have been attributed to cigarette smoking, ${ }^{2}{ }^{3}$ with age as an additional risk factor. Furthermore, age ${ }^{45}$ and smoking ${ }^{6} 7$ are strongly associated with comorbidity, that is, diseases and conditions coexisting with lung cancer. ${ }^{8}$ As our society ages, clinicians

\section{Strengths and limitations of this study}

- The strength of this study is that it is a population-based study.

- In this study, we used Charlson comorbidity index with only hospital diagnoses. It is, thus, possible that some patients with comorbid conditions may have been misclassified as having no comorbidity.

will encounter older patients more frequently and with increasing probability that patients with lung cancer will have coexisting diseases. It is well established that comorbidity has an effect on survival. ${ }^{9} 10$

However, comorbidity may influence survival in different ways. First, patients with lung cancer frequently present with other diseases, including chronic obstructive lung disease, cerebrovascular diseases, heart failure and myocardial infarction. Such types of comorbidity may by itself have a negative effect on survival. Second, comorbidity may significantly mask symptoms and delay the establishment of the diagnosis of lung cancer or even prevent a full diagnostic evaluation with proper staging of the disease. Third, surgical intervention has a positive effect on the survival of lung cancer, ${ }^{11}$ but comorbidity may contradict surgical intervention in patients otherwise eligible for surgery. "Mostly, comorbidities will have a negative impact on survival, but it can increase the person's contact with the medical practitioners as it may indirectly have a positive impact on survival by increasing the likelihood of earlier diagnosis." 
Simultaneous estimation of models describing a diagnostic process, surgical intervention, along with the survival process, makes more efficient use of available data and make it possible to estimate the influence of comorbidity with respect to diagnostic procedures, treatment options and the prognosis in patients with lung cancer in a situation with partially missing data. Since non-small cell lung cancer (NSCLC) and small cell lung cancer (SCLC) differ in the sense of clinical characteristics, treatment options and survival, this study was restricted to patients with NSCLC.

\section{METHODS}

Patient population and clinical data

The Danish Lung Cancer Registry

Since the establishment in 2000, the Danish Lung Cancer Registry (DLCR) has accumulated data on all cases of lung cancer as reported from about 50 departments involved in the care of primary lung cancer in Denmark. ${ }^{12}$ Data are reported to the database when the diagnostic evaluation has been completed, and when a specific treatment has been finished. This registry information is then supplemented with data on the patient's vital status retrieved from the Danish Civil Registration System, and pathology information related to the lung cancer case from the Danish Pathology Register.

\section{Diagnostic evaluation and treatment options}

Diagnostic procedures in suspected lung cancer are primarily performed to establish that the presence of disease and the type and clinical staging of lung cancer. Lung cancer is divided into two main types based on histology, SCLC (10-15\%), and NSCLC (85-90\%). When the type of lung cancer is established, further investigations are performed to evaluate whether the patient is eligible for treatment, and if so, what kind of treatment. Patients with lung cancer with NSCLC are, in principle, treated with surgical resection or chemotherapy and/or radiotherapy. Surgical resection of the tumour is associated with the most favourable survival rates, but only $20 \%$ of the patients are eligible for resection at the time of diagnosis. The clinical stage is the most important factor when deciding the choice of treatment. However, the true stage is only identifiable in connection with surgery. The risk of misclassification depends on how advanced the disease is at the time of diagnosis. Since it is relatively easy to stage a patient with an advanced disease (large tumour involving other organs and/or metastasis corresponding to clinical stage IIIb or IV), the risk of misclassification is of minor importance in an advanced disease. On the other hand, misclassification is more common in a local disease, where it can be difficult to distinguish between the denominators defining the different subcategories of clinical stages I-IIIa. Since the choice of treatment, to a great extent, depends on the clinical stage, misclassification in a local disease does affect the type of treatment offered to the patient. Furthermore, a range of other prognostic factors are also taken into account before the final decision about treatment is made, including age, alcohol or drug missuse and comorbidity.

\section{Comorbidity}

We included information on comorbidity for each patient up to 10 years before the lung cancer diagnosis, using the Danish National Patient Register, which was established in 1977. This register contains data including coding of all interventions related to diagnostic evaluation and treatment for all somatic patient admissions in Denmark. ${ }^{13}$ For the classification of comorbidity, we used a slight modification of the Charlson comorbidity index $(\mathrm{CCI})^{14}$ by excluding all interventions with lung cancer as the activity diagnosis and registered prior to the date of diagnosis for the present patient group (see below). This was carried out in order to avoid the contribution to the CCI from the very few patients who had a previously registered course of lung cancer in the Danish National Patient Register. Relevant diseases are grouped into a total of 19 categories, each of which assigned a score between 0 and 6 depending on assumed severity. The CCI is calculated as the sum across these categories and will range between 0 (with no diseases in the medical history qualifying for inclusion in the CCI) and 37 (a medical history representing all diseases of the highest severity, qualifying for inclusion in the CCI). As the Danish National Patient Register covers all somatic activities, all patients are identifiable without exceptions. Thygesen et $a l^{15}$ showed that the predictive value of using the coding practice in the register to establish the CCI is consistently high. Any hospital contact represented with a cancer diagnosis registered within 150 days before the date of lung cancer diagnosis was excluded from contribution to the CCI. This was carried out to avoid the influence of misclassification by cases with a cancer diagnosis (including cancers of neighbouring organs) that eventually turned out to be verified as lung cancer. Based on a sensitivity analysis, only very few, if any, cancer of other organs than the lungs will be missed by this procedure. Patients with lung cancer were, thereafter, grouped according to the increased level of CCI as follows: (1) persons with a CCI score of 0; (2) persons with a CCI score of 1-2 and (3) persons with a CCI score of $3+$.

\section{Study population}

We have chosen to base our analysis on a subset of DLCR, which consists of all 3135 patients with NSCLC who were registered in 2010. We have the information on age, sex, clinical stage, resection status and district on 2840 of those patients. The DLCR is described in detail in ref. 12.

The detailed distribution of CCI in the patient sample can be seen in figure 1 . The proportions of patients in three comorbidity groups are $46.4 \%, 38.1 \%$ and $15.5 \%$. 


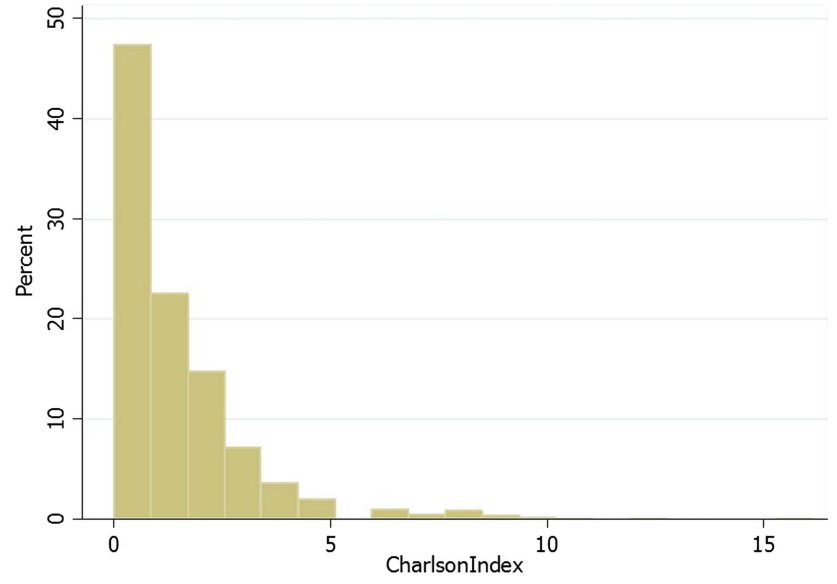

Figure 1 Distribution of Charlson comorbidity index in the study population.

Table 1 shows the relationship between clinical and surgical stages. Only $16 \%$ of the patients have a surgical stage registered. In this subset, the clinical and surgical stages are identical for $430(68 \%)$ patients, while in 127 $(20 \%)$ of the patients the clinical stages are classified as lower than the surgical stage and in $73(12 \%)$ of the patients the clinical stages are rated higher than the surgical stage.

\section{Model formulation}

For each individual, we observe survival data and covariate data. We assume the survival data to be subject to right censoring. For each individual, there are fully or partially observed vector of confoundings consisting of age, sex, comorbidity, clinical stage, surgical stage and resection status. There are five districts in Denmark in total. Recently, heterogeneity across Danish districts in the survival of patients with lung cancer has been demonstrated. ${ }^{16}$ This heterogeneity cannot be ignored, and thus districts will be treated as dummy variables in the models (see figure 2).

Our proposed method consists of three models. The first one describes the likelihood model for the resection status in the form of a logistic regression adjusted for age, sex, comorbidity and clinical stage. We hereafter refer to this model as the 'resection model'.

Table 1 Distribution between clinical and surgical stages in the study population

\begin{tabular}{|c|c|c|c|c|c|c|c|}
\hline \multirow{2}{*}{$\begin{array}{l}\text { Clinical } \\
\text { stages }\end{array}$} & \multicolumn{5}{|c|}{ Surgical stages } & \multirow{2}{*}{$\begin{array}{l}\text { No data } \\
\text { (no surgery) }\end{array}$} & \multirow[b]{2}{*}{ Total } \\
\hline & $\overline{0, I}$ & II & Illa & IIIb & IV & & \\
\hline 0,1 & 280 & 56 & 28 & 0 & 4 & 187 & 555 \\
\hline II & 39 & 98 & 33 & 1 & 5 & 153 & 329 \\
\hline Illa & 8 & 16 & 33 & 1 & 0 & 374 & 431 \\
\hline IIlb & 0 & 1 & 0 & 1 & 0 & 371 & 373 \\
\hline IV & 3 & 2 & 1 & 0 & 13 & 1489 & 1508 \\
\hline Total & 330 & 173 & 95 & 3 & 22 & 2573 & 3196 \\
\hline
\end{tabular}

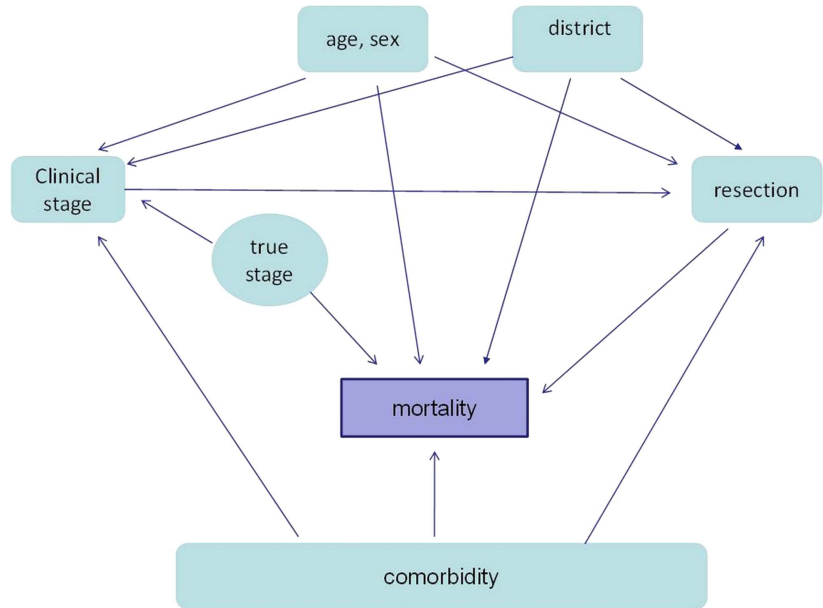

Figure 2 Graphical representation of the model.

The second model describes the likelihood model for the surgical stage in the form of an ordinal logistic regression adjusted for age, sex, comorbidity and true stage. We hereafter refer to this model as the 'staging model'.

The last model is a survival model. Here we estimate the hazard of failure through the proportional Cox regression model, ${ }^{17}$ where the hazard depends on the covariate through its current value adjusting for age, sex, comorbidity, resection status and true stage. We hereafter refer to this model as the 'survival model'. Here we used a sandwich estimator derived by Lin and Wei. ${ }^{18}$ Lin and Wei show that the estimate is consistent and robust to several possible misspecifications in the Cox model including the lack of proportional hazard and incorrect functional form for the covariates.

To estimate the direct and indirect effect of comorbidity on survival, these three models must be estimated jointly in one simultaneous procedure.

\section{Assumptions}

Surgery provides for the optimal possibility of correct disease staging of the patient. Therefore, in our notation, the true stage is equal to the surgical stage.

As aforementioned, the true stage is observed only for the patients who have had surgery, which is less than $20 \%$ of all patients. In this study, we assume that the classification process for patients without surgery is identical with that for patients with surgery, that is, the missing data process for observing 'true stage' is missing at random. Using this assumption, we can handle the missing data problem using one of the common techniques for this purpose: multiple imputation.

\section{Framework for multiple imputation}

Generally, there are three mechanisms behind missing data: ${ }^{19}$ data can be 'Missing Completely at Random' (MCAR), 'Missing at Random', (MAR) and data can be missing in an unmeasured fashion 'Missing Not at Random' (MNAR). See refs. 20 and 21 for review of important statistical methods for missing data. 
We assume the missing data in our sample to be MAR. Imputation and weighting ${ }^{22} 23$ are two important approaches in dealing with MAR missing data problems. Wang and coauthors ${ }^{2}$ show that in many situations, some inverse selection probability-weighted estimators are numerically equivalent to imputation. The performance of multiple imputation has been well studied and it has been shown to perform favourably. ${ }^{25-27}$ If MAR holds, it has been shown that multiple imputation produces unbiased parameter estimates which reflect the uncertainty associated with estimating missing data. Moreover, multiple imputation has been shown to be robust to departures from normality assumptions. ${ }^{28}$

There are many different ways to impute values, constructing a complete dataset. In this work, we use the stochastic regression imputation. Missing values were replaced by predicted values from a regression modelcontained covariates: age, sex, comorbidity and clinical stage plus residuals, drawn to reflect uncertainty in the predicted values.

According to King et $a l,{ }^{29}$ about 5 or 10 imputed datasets are often satisfactory. In Bayesian simulation, the distribution of variables in missing data process simulated jointly as well as parameters in a regression equation, that is, in WinBugs ${ }^{30}$ (estimation platform for Bayesian simulations), the programme is going to treat all of the missing elements of the data as if they were unknown model parameters.

\section{RESULTS}

Table 2 shows the descriptive characteristics of the study population.

Table 3 shows the estimated effect of comorbidity and other adjusting parameters. First, consider the results for the resection model. The model shows that the increasing level of comorbidity significantly reduces the probability of resection. Models also show that increasing age reduces the probability of resection; sex has no statistically significant effect on the probability of resection; a

Table 2 Descriptive characteristics of the study population

\begin{tabular}{lc}
\hline & $\mathbf{N}(\%)$ \\
\hline Age & \\
$<67)$ & $1209(42.6)$ \\
$\geq 67$ & $1631(57.4)$ \\
Sex & \\
Male & $1467(51.6)$ \\
Female & $1373(48.4)$ \\
Operation & \\
Yes & $540(19)$ \\
No & $2300(81)$ \\
CCI & \\
0 & $1318(46.4)$ \\
1 & $1082(38.1)$ \\
$>1$ & $440(15.5)$ \\
\hline
\end{tabular}

Table 3 Estimating results of the combination of models based on 2000 Monte Carlo dataset simulations reported by HR with $95 \%$ credible interval for the Cox regression (Survival model) and OR with 95\% credible interval for the logistic regression (resection model) and for the ordered logistic regression (classification model)

\begin{tabular}{lll}
\hline Model & Parameter & $\begin{array}{l}\text { HR/OR } \\
(\mathbf{2 . 5 \%} ; 97.5 \%)\end{array}$ \\
\hline Resection & Age $(\geq 67$ vs $<67)$ & $0.47(0.36 ; 0.63)$ \\
& Sex (female vs male) & $1.01(0.79 ; 1.35)$ \\
& Comorbidity (growing) & $0.65(0.54 ; 0.79)$ \\
Classification & Clinical stage (growing) & $0.19(0.16 ; 0.21)$ \\
& Age $(\geq 67 v s<67)$ & $0.53(0.47 ; 0.66)$ \\
& Sex (female vs male) & $1.23(1.08 ; 1.49)$ \\
& Comorbidity (growing) & $1.08(0.96 ; 1.20)$ \\
Survival & True stage (growing) & $0.41(0.38 ; 0.45)$ \\
& Age ( $\geq 67$ vs $<67)$ & $1.30(1.16 ; 1.40)$ \\
& Sex (female vs male) & $0.87(0.78 ; 0.93)$ \\
& Comorbidity (growing) & $1.08(1.02 ; 1.14)$ \\
& Resection status (yes vs & $0.17(0.15 ; 0.21)$ \\
& no) & \\
& True stage (growing) & $0.13(0.08 ; 0.16)$ \\
\hline
\end{tabular}

high clinical stage reduces the probability of resection substantially.

The staging model is most influenced by the missing data process and the staging model shows, as expected, that the true stage is negatively correlated with the clinical stage. The model also indicates that age and sex have an influence on the staging process. Moreover, it shows that increasing comorbidity has a slight, but not significant, effect on the staging process.

The survival model shows that increased comorbidity increases the mortality significantly. Increased age and advanced clinical stage are associated with the significantly increased mortality. Women have a significantly better survival compared with men. Resection is associated with a substantial reduction in mortality.

In addition, we performed an analysis with an alternative assumption about the missing data process, namely, that there is no misclassification of stages for patients without surgery. In that case, the measurement of clinical stage was used in models (2) and (3) instead of true stage, for the patients without surgery. The results of both analyses are very similar with respect to direct and indirect effects of comorbidity on survival.

\section{DISCUSSION}

In this paper, we used an estimation method that allows a combination of different models in order to estimate the direct and indirect impact of comorbidity on survival in a situation with partially incomplete data.

In our study, the missing data problem concerns the lack of information on the true stage in patients who have not had surgery. We manage this problem by applying assumptions that represent two clinically extreme 
scenarios, "no misclassification at all for patients without resection' and 'misclassification at random'. We cannot be certain which scenario is closer to the reality, but clinical experience suggests that the real misclassification process for the patients, who were not operated, is somewhere in between these two scenarios. In clinical practice, it is well known that treatment with the intention of cure, such as resection, requires precise pretreatment patient evaluation including valid clinical staging. Owing to this, it is plausible that misclassification in this group of patients is smaller than in patients selected for a palliative treatment. On the other hand, it is often easier and faster to come to a diagnostic conclusion in patients with advanced disease, and decisions about treatment are, therefore, made before all investigations are finished, thus making the staging more uncertain. Despite the fact that we used two clinically opposite assumptions about the missing data process, the direct effect of comorbidity on the estimated survival, using both approaches, is substantially equal. This may be because our model is quite stable, but could also be explained by bias in both estimates. Further work is needed to clarify this.

In this study, the variable resection was treated as known at baseline. We are aware that it potentially could be a source of bias. We believe that this bias is disparaged to be small, and therefore could be ignored. As all the information needed to decide about resection is presented at the baseline and mortality in the group of potentially inoperable patients is very small, in the period from baseline (day of diagnose) to operation day.

From a clinical point of view, our results seem plausible. The estimated effects of age, sex, stage and resection are generally as expected concerning the probability of resection, staging and survival. It appears that the direct and indirect effects of comorbidity in general are as expected.

In this study, we used CCI with only hospital diagnosis of diseases as a measure of comorbidity. It is, thus, possible that some patients with comorbid conditions may have been misclassified as having no comorbidity. It will be relevant to perform the same analysis using CCI based on diagnoses from general practice; unfortunately, these data are not available yet. In the future work, we will investigate the prognostic effect of the individual diseases contributing to the overall CCI on the survival of patients with lung cancer.

We conclude that our work represents a useful solution to the statistical management of the complex influence of comorbidity on survival under incomplete data. We have used NSCLC, but the approach seems applicable to other diseases with similar complexity. The proposed approach can be easily generated to other applications.

\section{CONCLUSION}

We found that comorbidity has a significant indirect effect on survival of NSCLC patients mediated by the resection process, and a slightly direct effect on mortality. Further research is needed to compare the performance of the CCI to other comorbidity indices.

\section{Author affiliations}

${ }^{1}$ Center for Clinical Epidemiology, Odense University Hospital and Research

Unit of Clinical Epidemiology, Institute of Clinical Research, University of

Southern Denmark, Odense, Denmark

${ }^{2}$ Odense Patient data Exploratory Network (OPEN), Institute of Clinical Research, University of Southern Denmark, Odense, Denmark

${ }^{3}$ Department of Thoracic Surgery, The Danish Lung Cancer Registry, Odense University Hospital, Odense, Denmark

Acknowledgements The authors would like to thank Peter Gustav, academic data manager, for establishing the algorithm to calculate Charlson Comorbidity Index from the Danish Patient Registry.

Contributors $\mathrm{MI}$ and AG conceived the study idea and designed the study. MI led the statistical analysis. All authors participated in the discussion and interpretation of the results.

Funding This research received no specific grant from any funding agency in the public, commercial or not-for-profit sectors.

\section{Competing interests None.}

Provenance and peer review Not commissioned; externally peer reviewed. Data sharing statement No additional data are available.

Open Access This is an Open Access article distributed in accordance with the Creative Commons Attribution Non Commercial (CC BY-NC 3.0) license, which permits others to distribute, remix, adapt, build upon this work noncommercially, and license their derivative works on different terms, provided the original work is properly cited and the use is non-commercial. See: http:// creativecommons.org/licenses/by-nc/3.0/

\section{REFERENCES}

1. Hakulinen T, Engholm G, Gislum M, et al. Trends in the survival of patients diagnosed with cancers in the respiratory system in the Nordic countries 1964-2003 followed up to the end of 2006. Acta Oncol 2010;49:608-23.

2. Shopland DR, Eyre HJ, Pechacek TF. Smoking-attributable cancer mortality in 1991: is lung cancer now the leading cause of death among smokers in the United States? J Natl Cancer Inst 1991;83:1142-8.

3. Siemiatycki J, Krewski D, Franco E, et al. Associations between cigarette smoking and each of 21 types of cancer: a multi-site case-control study. Int J Epidemiol 1995;24:504-14.

4. Yancik R. Cancer burden in the aged: an epidemiologic and demographic overview. Cancer 1997;80:1273-83.

5. Yancik R. Epidemiology of cancer in the elderly. Current status and projections for the future. Rays 1997;22:3-9.

6. Doll R, Peto R, Wheatley K, et al. Mortality in relation to smoking: 40 years' observations on male British doctors. BMJ 1994;309:901-11.

7. Peto R, Lopez AD, Boreham J, et al. Mortality from smoking worldwide. Br Med Bull 1996;52:12-21.

8. Tammemagi CM, Neslund-Dudas C, Simoff M, et al. In lung cancer patients, age, race-ethnicity, gender and smoking predict adverse comorbidity, which in turn predicts treatment and survival. J Clin Epidemiol 2004;57:597-609.

9. Extermann M. Interaction between comorbidity and cancer. Cancer Control 2007:14:13-22.

10. Firat S, Byhardt RW, Gore E. The effects of comorbidity and age on RTOG study enrollment in stage III non-small cell lung cancer patients who are eligible for RTOG studies. Int $J$ Radiat Oncol Biol Phys 2010;78:1394-9.

11. Jakobsen $\mathrm{E}$, Palshof $\mathrm{T}$, Østerlind $\mathrm{K}$, et al. Data from a national lung cancer registry contributes to improve outcome and quality of surgery: Danish results. Eur J Cardiothorac Surg 2009;35:348-52.

12. Jakobsen E, Green A, Oesterlind K, et al. Nationwide quality improvement in lung cancer care: the role of the Danish Lung Cancer Group and Registry. J Thorac Oncol 2013;8:1238-47.

13. Andersen TF, Madsen M, Jørgensen J, et al. The Danish National Hospital Register. A valuable source of data for modern health sciences. Dan Med Bull 1999;46:263-8. 
14. Charlson ME, Pompei P, Ales KL, et al. A new method of classifying prognostic comorbidity in longitudinal studies: development and validation. J Chronic Dis 1987;40:373-83.

15. Thygesen SK, Christensen CF, Christensen S, et al. The predictive value of ICD-10 diagnostic coding used to assess Charlson comorbidity index conditions in the population-based Danish National Registry of Patients. BMC Med Res Methodol 2011; 11:83.

16. Danish Lung Cancer Group. Årsrapport. 2012. http://lungecancer.dk/ 00007/00058/

17. Cox DR. Regression models and life-tables (with discussion). J R Stat Soc 1972;34:187-220.

18. Lin DY, Wei LJ. The robust inference for the Cox proportional hazard model. J Am Stat Assoc 1989;84:1074-8.

19. Little RJA, Rubin DB. Statistical analysis with missing data. 2nd edn. New York: John Wiley \& Sons, 2002.

20. Little RJA. Regression with missing X's: a review. J Am Stat Assoc 1992;87:1227-37.

21. Ibrahim JG, Chen H, Lipsitz SR, et al. Missing data methods for generalized linear models: a comparative review. J Am Stat Assoc 2005;100:332-46.

22. Robins JM, Rotnitzky A, Zhao LP. Estimation of regression coefficients when some regressors are not always observed. J Stat Assoc 1994;89:846-66.
23. Qi L, Wang CY, Prentice RL. Weighted estimators for proportional hazard regression with missing covariates. J Am Stat Assoc 2005;100:1250-63.

24. Wang CY, Lee SM, Chao EC. Numerical equivalence of imputing scores and weighted estimators in regression analysis with missing covariates. Biostatistics 2007;8:468-73.

25. Graham JW, Schafer JL. On the performance of multiple imputation for multivariae data with small sample size. In: Hoyle R. ed. Statistical strategies for small sample research. Thousand Oaks, CA: Sage, 1999:1-32.

26. Schafer JL, Graham JW. Missing data: our view of the state of the art. Psychol Methods 2002;7:147-77.

27. Taylor L, Zhou XH. Multiple imputation methods for treatment noncompliance and nonresponse in randomized clinical trials. Biometrics 2009;65:88-95.

28. Wayman JC. Multiple imputation for missing dta: What is it and how can I use it? Paper presented at the Annual Meeting of the American Educational Research Association; Chicago, IL, USA, 2003.

29. King G, Honaker J, Joseph A, et al. Analyzing incomplete political science data: an alternative algorithm for multiple imputation. $A m$ Political Sci Rev 2001;9:49-69.

30. Lunn DJ, Thomas A, Best N, et al. WinBUGS-a Bayesian modelling framework: concepts, structure, and extensibility. Stat Comput 2000;10:325-37. 